\title{
An Evaluation of Laser Cleaning as a Conservation Technique for an Archaeological Metal Coins
}

\author{
Mohammed Tharwat Mohammed Elshareef* \\ Conservation Department, Faculty of Fine Arts, Minia University, Egypt
}

\begin{abstract}
Preservation of cultural property for the future is one of the most important activities of our society. The information which we can gather from such materials is a valuable key to understanding the past. Recent developments in laser techniques in the conservation field have allowed us to test the laser cleaning, Although laser cleaning has gained acceptance in other areas of conservation (e.g. Stone statues and paintings) the process has not been widely applied to metal artifacts of historical value. The potential of laser techniques in conservation has needed a long development period to be fully demonstrated. The possibility to achieve a very precise and selective removal of deteriorated materials was implemented through a series of interdisciplinary studies focussed on laser ablation of specific materials .Therefore, the research will discusses and evaluate the use of laser as a modern technique for cleaning an archaeological coins with multiple experiments of another researches.
\end{abstract}

\section{Keywords:}

Metal-Coins-Laser-Archeology-Conservation

\section{Introduction}

Ancient metals have been studied in the past on a regular basis, with emphasis on the surface modifications they have suffered in the course of time[E. Drakaki ,2004],Choosing the appropriate safe technique depends on the original material being treated and the crust[Walid K. Hamoudi,2015], Laser cleaning represents the most important contribution of physics to the conservation of cultural heritage. Despite the pioneering works dating back to the early 1970s[S. Siano,2012], In recent years advances in technology have created tools that are revolutionizing conservation techniques. The latest of these is the use of high-intensity light (lasers) for surface cleaning of artifacts. Lasers remove/clean undesirable contaminants as well as old and decayed conservation materials to preserve the original material. The technology is based on the selective vaporisation of an optically absorbing substance such as a contaminant from the reflective underlying surface. During the past 30 years cleaning of different materials using laser radiation has been studied and compared with the other methods and has shown to give good results[Cooper M.,1998].

\footnotetext{
* Correspondent author : mtharwatm@yahoo.com
} 


\section{Conclusion}

\subsection{Using laser in Conservation of artifacts}

Lasers are sources of temporally and spatially coherent light, which results in light that is strongly monochromatic. This property, along with high intensity (due to beam collimation) and the possibility to generate short and ultra-short pulses, meaning high peak power, makes laser light especially suited for applications in surface and materials science[Carla Lucía,2016]

Nowadays lasers are used widely in analysis, monitoring, and conservation of cultural heritage objects . Their unique properties are being responsible for high control, selectivity, minimal contact, and versatility; attributes that are essential for any conservation intervention on such valuable objects. In metal conservation, there have been plenty of studies aiming to use lasers for the removal of different corrosion layers, encrustations, and coatings from various metal surfaces with mixed results[A. Siatou,2007]

\subsubsection{Types of laser used in cleaning}

There are many different types of lasers which have varying wavelength and energy characteristics. Lasers are unique sources of light, providing energy in the form of a very intense, monochromatic, well-collimated beam which offers many advantages over traditional methods. Laser cleaning is a selective, non-contact method that can lead to better preservation of the surface and surface details. Furthermore, laser cleaning is safer from the point of view of working conditions and the environment [Kautek W., König E.,1997].

TEA CO2 lasers have been successfully used for removal of organic materials and rust. Q-switched Nd:YAG lasers at IR and at green wavelengths, which have different absorption characteristics, have also been tested. The Nd:YAG laser was able to clean the corroded samples, however, the risk of surface damage was higher than when using the TEA CO2 laser.A comparison between laser cleaning and micro blasting revealed that the lasers achieved a superior performance[Y.S. Koh, I. Sárady.,2005].

\subsubsection{Mechanisms of laser cleaning}

Laser surface cleaning has become a mature technological process, widely applied in electronic, air, nuclear industry as well as artworks conservation The efficiency of the cleaning process depends on many factors such as characteristics of laser radiation, optical, mechanical and thermal properties of material under treatment[A. Sarzyński, K. Jach, et al.,2005]. Cleaning by laser irradiation involves complex mechanisms such as photo thermal, photochemical and mechanical effects on the target material. The exact mechanisms which are active depend on the parameters of the laser irradiation and on the physical and chemical properties of the surface[Yang Sook Koh., 
,2005], There are two types of laser cleaning: dry cleaning and steam one, which are based on the pulse laser heating of solid surface(dry) and in presence of liquid layer on it, correspondingly. By increasing laser pulse energy the method of dry cleaning turns into evaporative cleaning. The technique of dry cleaning is simple, but its effectiveness is less, than for steam cleaning, and required laser intensity is higher (for nanosecond pulses). Power thresholds of the steam cleaning are in 2 or 3 times higher than for dry cleaning. Sometimes dry laser cleaning can result in local damage of the surface. If the mentioned shortcomings of dry laser cleaning turn out to be significant, steam laser cleaning has to be used[V.P.Veikol, T.Ju.Mutin, et al .,2008], both using pulsed lasers, but either with or without the presence of a thin liquid film (usually water). These are sometimes referred to as dry or steam laser cleaning respectively. In dry laser cleaning most of the incident energy can be absorbed on a targeted surface allowing selective cleaning by control of the laser wavelength. This technique is useful when the substrate and contaminant layer or particles have different laser absorption characteristics causing the substrate or particles to be rapidly heated by the laser pulse leading to their ejection or vaporisation. However, in certain applications such as the removal of encrustations, better cleaning efficiency can be achieved by choosing a laser wavelength that is strongly absorbed by the surface together with a thin water film[Watkins, K.G.,1997], An important role in the process is played by the presence of liquid, e.g., water, at the cleaned surface which substantially increases, for different substrates, the efficiency of particle and encrustation removal. Usually porous structure of encrustation facilitates absorption of atmospheric gases and water. A large surface area of pores and, most of all, the presence of gases and water, change the physical properties of encrustation and beam propagation conditions:

- Often increase average absorption coefficient

- Laser radiation repeatedly refracts and reflects, causing interference and local increase of power density

- Gas in pores favour ionization, causing additional increase of radiation absorption[A. Sarzyński , K. Jach, et al.,2005]

Water brushed or sprayed onto the surface before the laser irradiation will penetrate into the contaminant layer where it will be rapidly heated by the laser leading to explosive vaporisation of the water. This results in efficient particle removal from the surface. However, in a typical laser-cleaning situation, the expected peak temperature of perhaps $370^{\circ} \mathrm{C}$ together with the high transient pressure due to the vaporisation of the water of up to a few hundred atmospheres can result in substrate damage, especially in rough or porous surfaces. However, this peak temperature exists only for a very short time, and most material surfaces show no sign of damage after a few pulses[Tam, A.C.,1992] , LIBS technique is based on the focusing of high-power laser pulses onto a sample surface leading to the creation of plasma composed of excited species which 
emit light. Collection of the plasma light, followed by spectral dispersion and detection, permits identification of the elements present in the sample using their characteristic spectral lines (qualitative analysis) and measurement of their intensities provides quantitative analysis[B.Salle, P.Mauchien, S. Maurice,2007]

\subsection{Cleaning metals Using an ND:YAG Laser}

Metals artefacts experience the action of corrosion and oxidation processes due to the many reactive agents present in the air, in the water and in the ground where these objects were kept for centuries. These are the cases of archaeological metals when they are recovered from the excavation sites, or the case of archaeological metals found under the sea. Today as well metal artefacts host in polluted environments develop typical oxidation and corrosion layers. To stabilise the state of conservation these layers have to be removed in order to apply protective coatings[Renzo Salimbeni., 2006]

For the purposes of the conservation of metal artifacts it is often necessary to remove surface oxide layers without damaging the metal below. Various mechanical and chemical surface treatments are available for the removal of surface corrosion and other contaminants[Y.S. Koh, J. Powell, et al., ,2005] There are many different kinds of cleaning methods and techniques to choose from including mechanical and chemical cleaning. However, the choice of cleaning process to use in conservation is frequently complicated by questions such as 'what will be removed?' or 'what will be preserved?'. This is because the distinction between optimum cleaning and over cleaning is often quite subtle[Yang Sook Koh., ,2005] (Figure 1).

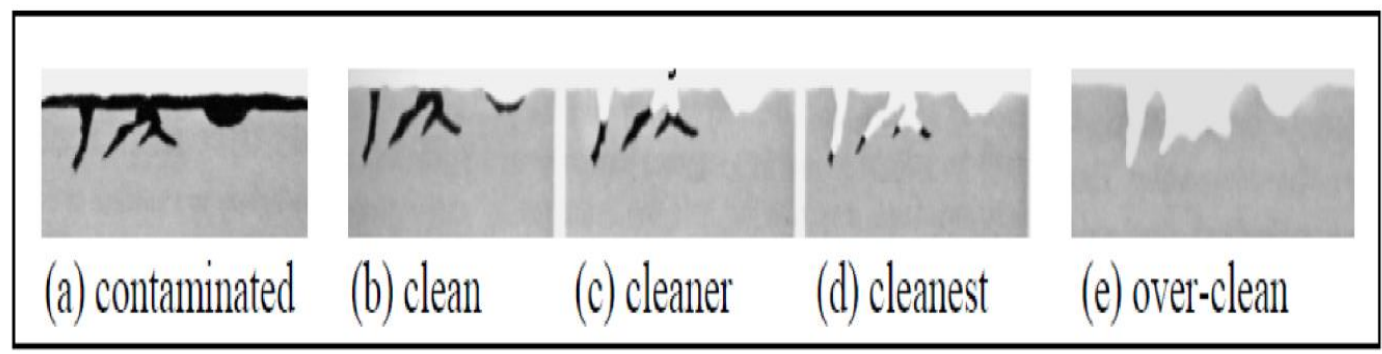

Figure 1. The distinction between optimum cleaning and over-cleaning is often quite subtle.

The conservator has to make a choice as to the final appearance of the treated object following the cleaning process[Yang Sook Koh., ,2005]

The restoration objective in cleaning metallic surfaces (focusing on copper, bronze, brass, silver), is uncovering the top (last) mineral surface belonging to the original, which becomes visible only after coats of paint, dirt and corrosion layers (e.g. copper sulphides and copper sulphates) not belonging to the original are removed. In doing so, as far as possible, the original surface of the work of art should not be altered by abrasion or chemical or physical processes, resulting in colour changes, loss of historical traces of work and polishing marks which accompany them[A. Gervais,2005]

The word Laser is an acronym for Light Amplification by Stimulated Emission of Radiation[Yang Sook Koh., ,2005], Since the invention of the first laser development 
has been extremely rapid and the unique properties of laser radiation have been applied in many different ways. Cleaning and removing of surface layers is one application where lasers can be used to generate clean surfaces without the use of environmentally hazardous solvents[Striebig, B.A.,2001], The first research studies on the application of laser technology to art restoration were performed in 1972 by Asmus and co- workers [Asmus, J.F., Murphy,1973]

\subsection{Laser cleaning of coins as positive and negative results}

Cleaning of artworks and other artefacts is desirable from an aesthetic point of view, but is often absolutely necessary to prolong their lifetime. In laser cleaning, laser irradiation is used for the removal of unwanted materials, such as pollutants, dirt, and corrosion products, in a precise, accurate, and highly controlled manner. Other advantages of the method include site selectivity, lack of mechanical or chemical contact with the substrate, and diversity in the types of substrates that can be treated. On the other hand, with commercially available lasers, some difficulties in the precise control of output power, and changes in beam profile, are encountered. This can result in surface damage due to uncontrolled melting or ablation[Carla Lucía,2016].

The research will review previous studies which used laser as positive and negative results of cleaning archaeological metal coins

\subsubsection{Positive result}

In this case[G Buccolieri ., et al.,2014] focus activity to study the laser cleaning of ancient bronze coins, undergone to corrosion laser cleaning is able to remove safely the patina film, i.e. corrosion products of the coin surface without damaging the coin matrix. In the area of a bronze coin irradiated by UV laser is showed. it is evident how the green colour of the patina disappears. The figure shows the bronze coin during the ablation process which subsequently affected the whole coin(Fig.2). Studies performed by using visible laser gave also good results on the removing of chlorine concentration from the surface of bronze coins, after 30 laser shots, The decreasing of tin signal reveals that visible laser cleaning is not selective because the removal of patina is accompanied by a reduction of tin.

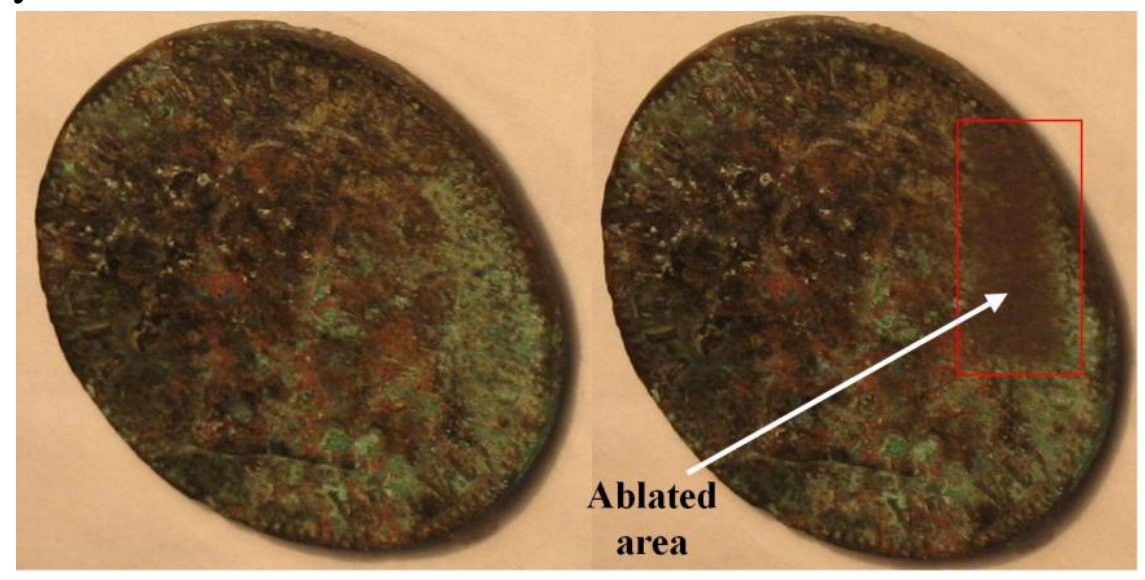


In another research Q-switched Nd:YAG laser at $1064 \mathrm{~nm}$ is very suitable technique for cleaning and investigating the ancient coins excavated from under Red Sea water archaeological site, Shoiba Port, Near Jeddah, Saudi Arabia. The results show that all contaminated elements were removed and evaporated from the tested coins after the laser cleaning. The best condition for cleaning and revealing the surface of a silver coin that has a medium layer of corrosion is the fifth shot (20 pulses). While for cleaning and revealing the surface of a silver coin that has a very thin layer of corrosion, the second shot ( 2 pulses only) is considered as the best condition to clean that coin. For using LIBS technique in analyzing ancient coins and evaluating the cleaning process, the number of shots of LIBS is a very important task while acquiring LIBS spectra. For the analysis of the corrosion products and other contamination elements on the surface of coin, the first shot is the best condition for acquiring the LIBS spectra. It means that the first shot is very useful for investigating the corrosion layer. The best shot for investigating the component of the core metal of the coin or the main composition of the coin depends on the thickness of the corrosion layers on the surface of the coin. In case that the coin has a medium layer of crusts, the fourth and the fifth shot are the best conditions for acquiring the LIBS spectra, while for the coin that has a very thin layer of tarnish, the second shot is the best condition for acquiring the LIBS spectra.[Omar Abdel-Kareem, Awad Al-Zahrani, et al.,2016]

Another study as a positive and negative results for using Laser, the potential of laser cleaning techniques in the conservation of ancient coins were investigated. The tests were carried out using different laser wavelengths of QS, LP, and FR Nd : YAG lasers, evaluating in each case which system appeared more appropriate. Laser cleaning with $x-y-z$ micro-adjustable stage could give excellent results, only when the silver alloy coins' surface is covered with an approximately thin and homogenous layer of corrosion products or when the corrosion products are more fragile, friable and powdery in relation to the patina condition, especially in the case of copper alloy coins. Laser cleaning, via an optical fiber beam delivery system could give satisfactory and successful cleaning effect, both in the case of homogeneous corrosion layer or in the case of corrosion heterogeneities and irregular soil deposits locally. Evidently, in the latter case, the microscopy approach could be more appropriate (Fig. 3). By using special laser microscope objective lenses, filters and a digital monitor, it could be possible to focus a laser beam on small areas of interest. Laser cleaning had no effect in removing the soil remains depositions on copper alloy coins. In addition, the outcome of laser application was not always successful, producing in some cases side effects, such as 
changes in the colour, in the chemical composition of corrosion products and thermal results[E. Drakaki, a B. Klingenberg, et al.,2010].
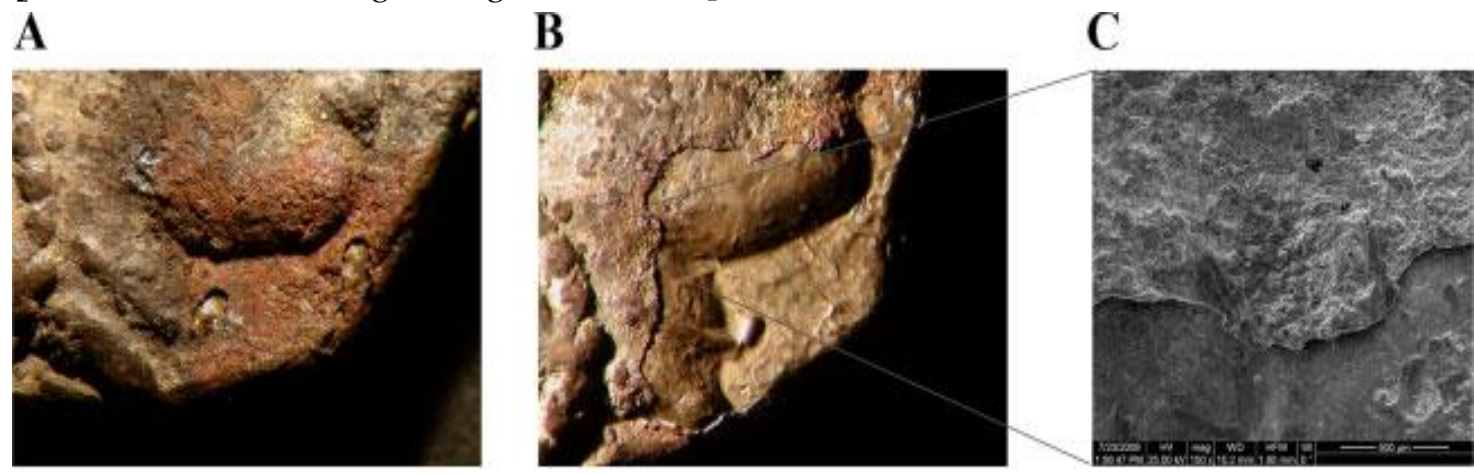

(Fig. 3) Optical and SEM microscopy photos of the surface morphology of laser cleaned area of coin.

A. During laser cleaning LQS Nd:YAG, .

B. After laser cleaning: $L Q S N d: Y A G$

C. SEM image. The two grey lines indicate the area where SEM image was taken

[E. Drakaki,a B. Klingenberg, et al.,2010].

Q-switched Nd:YAG laser at $1064 \mathrm{~nm}$ is very suitable technique for cleaning and investigation of ancient coins excavated from burial soil such as Najran excavation site. The results show that all elements of corrosion products that cover the tested coins were removed from the coins after the laser cleaning.( Figure 4) This will preserve the coins against the deterioration as cleaning the coin avoid the reactivation of corrosion processes. 30 pulses are the best condition for cleaning and reveal of the surface of silver coin that has a thick layer of corrosion. While 10 pulses are the best condition for cleaning and reveal the surface of silver coins that have a thin layer of corrosion such as the coin cleaned mechanically. For using LIBS technique in analysis of ancient coins and evaluating the cleaning process, the number of shots of LIBS is very important task for acquiring LIBS spectra. For analysis of the corrosion products, crusts and other elements of the corrosion products on the surface of coin, the first shot is the best condition for acquiring the LIBS spectra of the crust. It means that the first shot is very useful for investigation the corrosion and dirt layer. The best shot for investigation the component of the core metal of the coin or the main composition of the coin depends on the thickness of the crusts and the corrosion layers on the surface of the coin. In case that the coin has a thick layer of crusts, the fourth and the fifth shot are the best condition for acquiring the LIBS spectra. While for the coin that have a thin layer of tarnish, the second shot is the best condition for acquiring the LIBS spectra.[ Omar Abdel-Kareem, Awad Al-Zahrani, et al .,2016] 

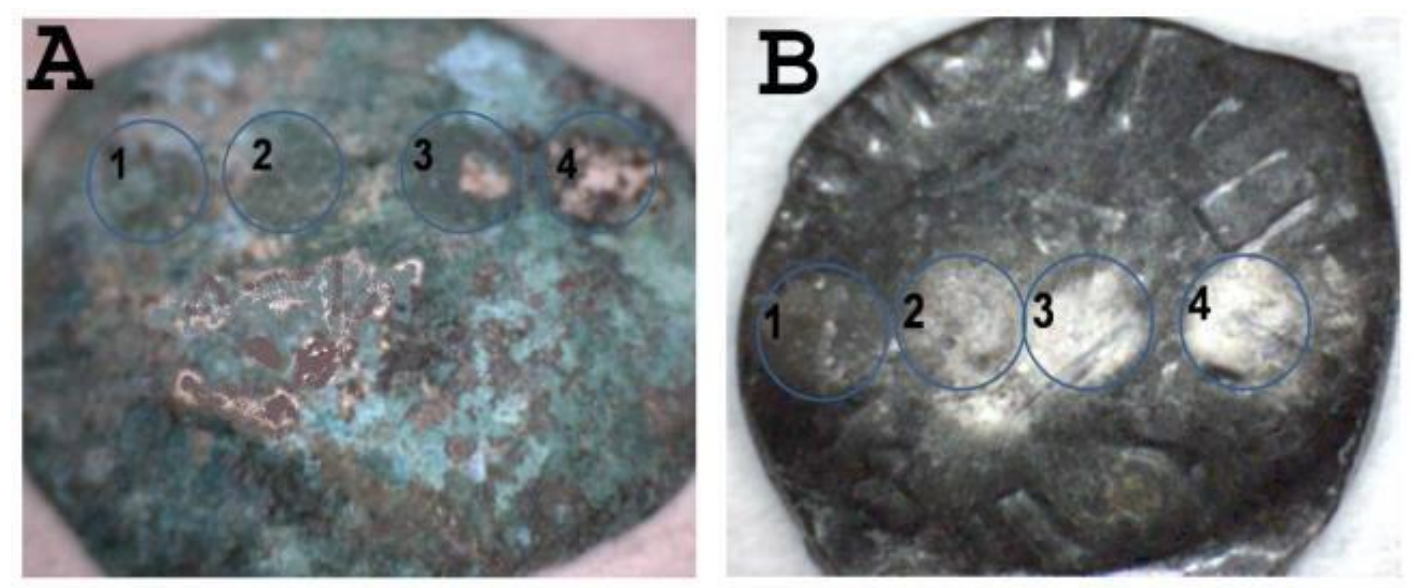

Figure 4: Process of laser cleaning of the studied corroded coins A) The corroded coin without any treatment before laser cleaning (1:1pulse, 2: 5 pulses, 3:15 pulses, 4:30 puls-es), B) The corroded coin after mechanical cleaning (1: 1 pulse, 2: 5pulses, 3: 10 pulses, 4: 20 pulses) [ Omar Abdel-

Kareem, Awad Al-Zahrani, et al .,2016]

\subsubsection{Negative results:}

In this study The green laser appeared to have a very damaging effect on the silver, removing the concretions but altering the appearance of the silver surface which looked more porous, less shiny and much less compact; it also destroyed some of the detail. The IR laser was much less damaging, but the cleaning still appeared a bit too harsh. The surface of the silver itself was superficially altered by the use of the laser, whether or not water was used. It acquired a whitish coloured (Fig.5) bloom, and this in turn also obscured the detail[M. Davis.,2005]
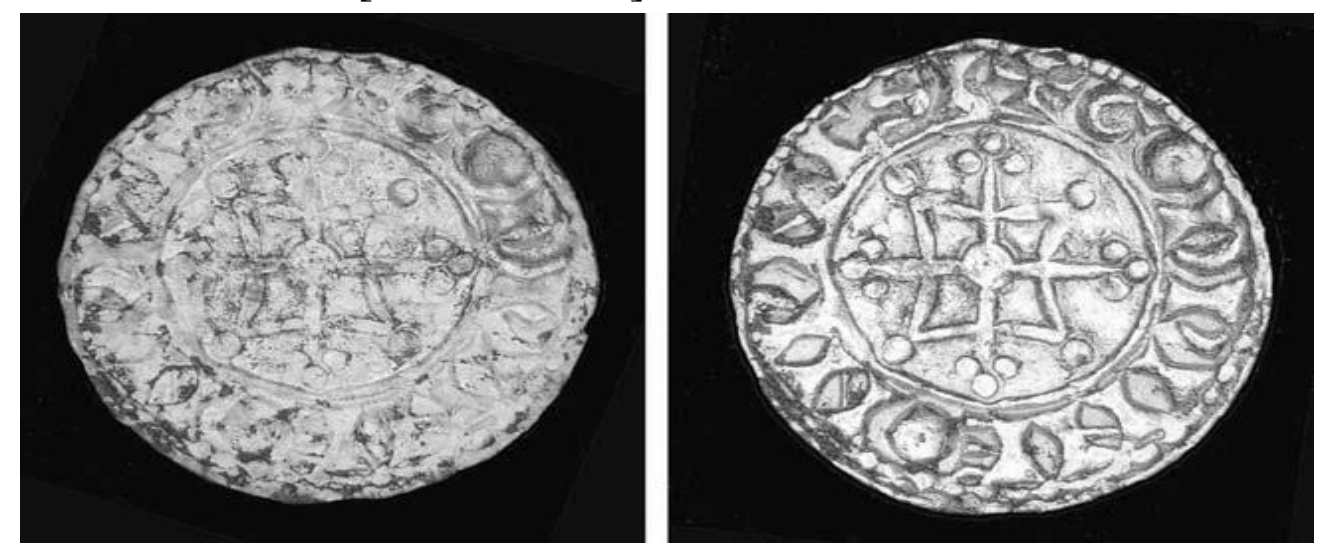

(Fig. 5) after laser cleaning showing (left) the cream coloured

bloom on the surface and (right) the cleaned coin after the bloom had been removed[M. Davis.,2005]

The laser was most successful at removing iron concretions adhering directly to the silver surface. It also removed green copper corrosion products from the coins where this had occurred. It did not remove silver sulphide so well. This was only present on a few of the specimens, but as it had occurred as a direct result of corrosion of the silver itself, the metal in these areas had a slightly rougher, pitted surface. The laser was not able to remove all the darker corrosion from indents and pores, and a greyish sheen was left on some of the coins[M. Davis.,2005]. 
In another case Laser cleaning tests were performed on five archaeological copper alloy objects using a Q switched Nd:YAG laser at $1064 \mathrm{~nm}$. As a comparison, a section of each object was cleaned mechanically. Prior to cleaning, cross-sections were prepared to characterise the corrosion crust and help to locate the position of the original surface. Laser cleaning was not successful at removing burial deposits on two of the objects. For the other three objects, the laser removed most of the corrosion crust. This was not always satisfactory, as cleaning was sometimes accompanied by the loss of the original surface. In addition, laser-cleaned surfaces were matt compared to mechanically cleaned surfaces. In some instances, the former had a disfiguring purple hue which was attributed to the formation of particles that could be seen when examining the surface using scanning electron microscopy. [Capucine Korenberg and Alexandra Baldwin.,2006].
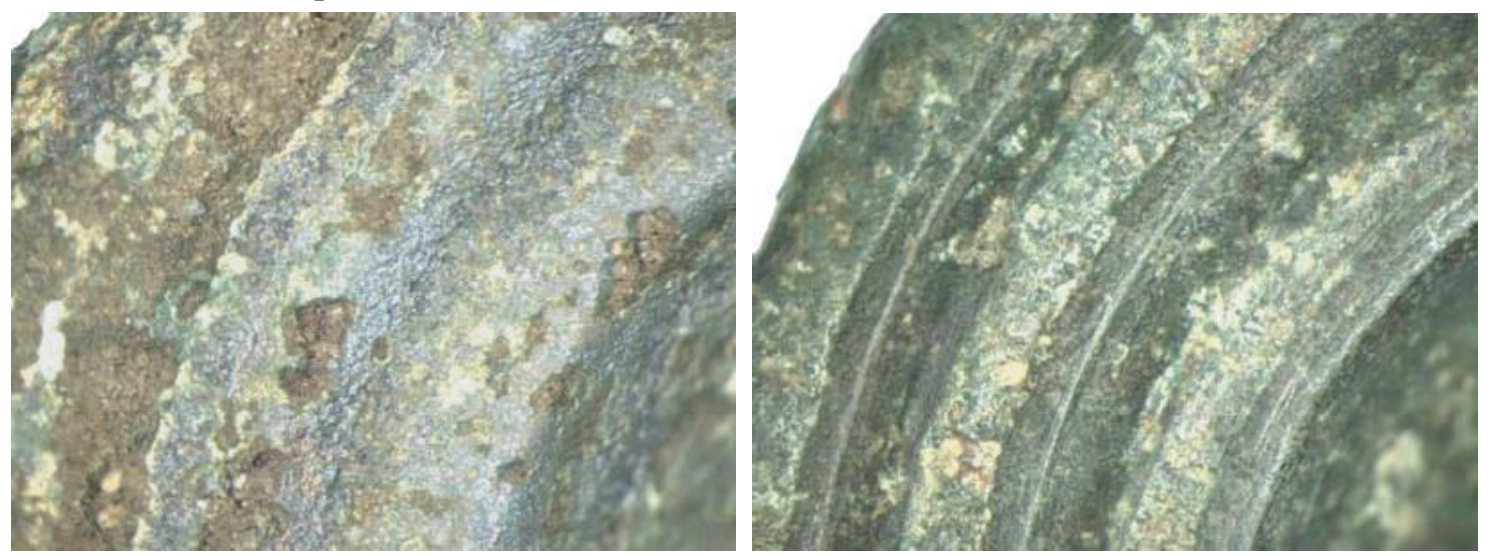

(Figure 6) Cleaning results for Object 4: (left) laser-cleaned surface

(right) mechanically cleaned surface [Capucine Korenberg and Alexandra Baldwin.,2006]

\section{Conclusion:}

The use of laser in the cleaning of metals in general and archaeological coins in particular has two results through previous studies related to the subject as there is research indicated the role of laser as a positive and other research has adversely affected the impact and from the point of view of the researcher must be done more studies to use laser on archaeological coins, For the thermal negative effects of the laser method . 


\section{References}

A. Siatou, D. Charalambous, et al., "A Comprehensive Study for the Laser Cleaning of Corrosion Layers due to Environmental Pollution for Metal Objects of Cultural Value: Preliminary Studies on Artificially Corroded Coupons" Laser Chemistry · January (2007) , p.1.

A.Gervais, M. Meier, et al., "Cleaning Historical Metals: Performance of Laser Technology in Monument Preservation" Lasers in the Conservation of Artworks, LACONA VI Proceedings, Vienna, Austria, Sept. 21-25, (2005) ,p.39.

A.Sarzyński , K.Jach, et al., "Comparison of Wet and Dry Laser Cleaning of Artworks" Lasers in the Conservation of Artworks, LACONA VI Proceedings, Vienna, Austria, Sept. 21-25,( 2005),p.161.

Asmus, J.F., Murphy, C.G. and Munk, W.H., "Studies on the interaction of laser radiation with art artifacts" Laser Technology II, SPIE Proceedings Volume 41, Bellingham, USA,(1973).

B.Salle, P.Mauchien, S. Maurice, “ Laser-Induced Breakdown Spectroscopy in open-path configuration for the analysis of distant objects”. Spectrochimica Acta Part B: Atomic Spectroscopy,62(8) (2007) p. 739.

Capucine Korenberg and Alexandra Baldwin., "Laser Cleaning Tests on Archaeological Copper Alloys Using an ND:YAG Laser" Hindawi Publishing Corporation, Laser Chemistry, (2006) pp.1-7.

Carla Lucía Soto Quintana., “ Development and optimisation of protocols for surface cleaning of Cultural Heritage metals" Erasmus mundus master in archaeological materials science, University of Évora, Évora, Portugal, September, (2016), PP.17-18.

Cooper M., "Laser Cleaning in Conservation An Introduction" Butterworth-Heinemann, Oxford,(1998).

E. Drakaki, A.G. Karydas, et al., “Laser cleaning on Roman coins” Applied Physics A 79 Materials Science \& Processing ,(2004),p.1111.

E. Drakaki,a B. Klingenberg, et al., "Evaluation of laser cleaning of ancient Greek, Roman and Byzantine coin " Surface Interface Analysis. 42, (2010),pp.671-674

G Buccolieri et al., "Analysis of selective laser cleaning of patina on bronze coins" Plasma Physics by Laser and Applications, ,Journal of Physics: Conference Series , Conference Series 508,(2014),pp.4-5

M. Davis., "Laser Cleaning the Abergavenny Hoard: Silver Coins from the Time of William the Conqueror" Lasers in the Conservation of Artworks , Lacona VI Proceedings, Vienna, Austria, Sept. 21-25,( 2005),p.47-51.

Omar Abdel-Kareem, Awad Al-Zahrani, et al ., "Evaluating laser cleaning of corroded archaeological silver coins" Mediterranean Archaeology and Archaeometry, Vol. 16, No 1,(2016), pp. 135-143

Omar Abdel-Kareem, Awad Al-Zahrani, et al., "evaluating the use of laser in analysis and cleaning of the Islamic marine archaeological coins excavated from the Red Sea" International journal of conservation science, Volume 7, Issue 2, April-June 2016: 511-522, May,(2016),p.520.

Renzo Salimbeni., "Laser techniques for conservation of artworks" Archeometriai Mühely,( 2006/1),p.37. 
S. Siano, J. Agresti, I. Cacciari ,et al., "Laser cleaning in conservation of stone, metal and painted artifacts: state of the art and new insights on the use of the Nd:YAG lasers" Journal of Modern Physics , (2012) 106, p.419.

Striebig, B.A., Roberts, T.L., et al., "Characterization of Emissions From Laser Removal of Surface Coatings" Conference of Laser Materials Processing, ICALEO, Jacksonville, (2001).

Tam, A.C., "laser-cleaning techniques for removal of surface particulates" Journal of Applied Physics, Vol.71, No.7, (1992), pp3515-3523.

V.P.Veiko1, T.Ju.Mutin, et al .,"Laser Cleaning of Metal Surfaces: Physical Processes and Applications", The International Society for Optical Engineering · January, (2008)

Walid K. Hamoudi, Mehdi S. Edan, et al ., " Laser surface cleaning of stones and some metal objects" The 5th International scientific Conference on Nanotechnology\& Advanced Materials Their Applications 3-4 Nov, (2015) ,p.973.

Watkins K.G., "A review of materials interaction during laser cleaning in art restoration" in: Kautek W., König E. (Eds.) "Lasers in the Conservation of Artworks" (LACONA I), Restauratorenblätter (Special Issue), Mayer \& Comp., Vienna, (1997), p.7-15.

Watkins, K.G., "A review of materials interaction during laser cleaning in art restoration" In Kautek W., König E. (eds.) Lasers in the Conservation of Artworks (LACONA I), Restauratorenblätter (Special Issue), Mayer \& Comp., Vienna, (1997), pp7-15.

Y.S. Koh , I. Sárady., "Cleaning of corroded iron artifacts using pulsed TEA CO2- and Nd:YAGlasers" Division of Manufacturing Systems Engineering Department of Applied Physics and Mechanical Engineering Luleå University of Technology Luleå, Sweden, December, (2005) ,p.33.

Y.S. Koh, J. Powell, et al., "Laser Cleaning of Corroded Steel Surfaces: A Comparison with Mechanical Cleaning Methods" Lasers in the Conservation of Artworks, LACONA VI Proceedings, Vienna, Austria, Sept. 21-25,( 2005),p.13.

Yang Sook Koh., "Laser Cleaning as a Conservation Technique for Corroded Metal Artifacts" Ph.D., Division of Manufacturing Systems Engineering Department of Applied Physics and Mechanical Engineering Luleå University of Technology Luleå, Sweden, December, (2005),pp.7-11. 